\title{
Eparanasi: un nuovo biomarker di fibrosi e un potenziale target farmacologico per ridurre la progressione del danno renale cronico
}

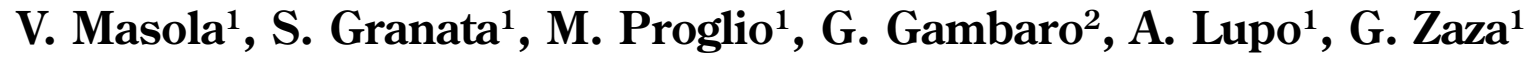 \\ ${ }^{1}$ Sezione di Nefrologia, Dipartimento di Medicina, Università di Verona, Verona \\ ${ }^{2}$ Unità di Nefrologia e Dialisi, Complesso Integrato Columbus-Policlinico Gemelli, Università Cattolica del \\ Sacro Cuore, Roma
}

\begin{abstract}
HEPARANASE AND RENAL FIBROSIS
Aвstract. The multiple drug therapy has led, over the years, a significant slowing of the chronic kidney disease progression towards end stage renal disease, but we are still far from the development of therapeutic interventions able to stop this mechanism. Clinical-pathological studies have clearly shown that fibrosis is the main process involved in chronic renal damage and that pathogenic mechanism underlying this condition arises in the tubular compartment. In particular, the epithelial-mesenchymal transition (EMT) plays an important role in the development of chronic damage. During EMT, the tubular epithelial cells acquire mesenchymal properties, undergo cytoskeleton remodeling and basement membrane degradation. Then they are able to migrate into interstitium where they play a key role in the fibrosis. In this context, seems to play a central role the enzyme heparanase (HPSE), an endo- $\beta$-D-glucuronidase that cleaves heparan sulfate (HS) side chains at a limited number of sites, hence participates in extracellular matrix (ECM) degradation and remodeling. The degradation of several constituents of the ECM, including heparan sulfate proteoglycans (HSPG), promotes the release of growth factors such as Fibroblast growth factor-2 (FGF-2) that induces the expression of mesenchymal markers alphaSMA, Vimentin (VIM) and fibronectin (FN), leads to degradation of the basement membrane by means of the secretion of matrix metalloproteinases (MMPs) and increases the cell motility. The heparanase expression is finely regulated by transcription factor, DNA methylation and various endogenous molecules. In vivo studies have shown its overexpression in histological sections of biopsies performed in patients with chronic kidney disease (e.g. diabetic nephropathy). Therefore, given the important role of this enzyme clinicians and researcher are developing several strategies to inhibit its gene expression and/or its enzymatic activity. Finally, it has been proposed its possible use as a biomarker of progression of tubulointerstitial damage for routinely use in clinical nephrology.
\end{abstract}

Key Words. Fibrosis, Chronic kidney disease, Epithelial-mesenchymal transition, Nephrology

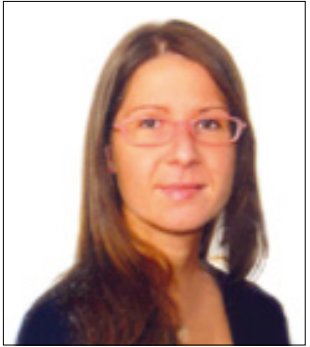

Valentina Masola

\section{Introduzione}

Lincidenza dell'insufficienza renale cronica (IRC) è in costante aumento nella popolazione occidentale e una significativa parte dei soggetti affetti da questa condizione clinica evolve verso l'uremia terminale con necessità di inizio del trattamento sostitutivo dialitico (emo- e peritoneo-dialitico) o l'effettuazione del trapianto renale (1). Pertanto, un adeguato inquadramento clinico e l'attuazione di programmi efficaci di prevenzione e/o di trattamento precoce dei fattori favorenti l'instaurarsi delle comorbidità associate riveste un ruolo importante nel migliorare la qualità di vita di questi soggetti, aumentare la sopravvivenza d'organo/pazienti e ridurre le spese di gestione da parte del Sistema Sanitario Nazionale.

Il trattamento poli-farmacologico (per esempio, inibitori del sistema renina-angiotensina-aldosterone) ha determinato, nel corso degli anni, un significativo mi- 
glioramento degli outcome clinici (2), ma siamo ancora distanti dallo sviluppo di interventi terapeutici in grado di rallentare drasticamente la progressione della catena patologica che porta alla perdita funzionale del rene.

Studi clinico-patologici hanno chiaramente mostrato che il principale elemento coinvolto nel danno cronico è la fibrosi tubulo-interstiziale e che una sua stima rappresenta un affidabile marker prognostico di progressione della malattia renale cronica (MRC) (3). Nel complesso questa condizione è semplicisticamente considerata un processo di cicatrizzazione dello spazio tubulo-interstiziale che insorge dopo il fallimento dei meccanismi fisiologici di risposta e rigenerazione tissutale dopo vari insulti acuti o in seguito al persistere di fattori di alterazione cronica (4). La progressiva deposizione di tessuto connettivo e l'infiltrazione di mio-fibroblasti nel parenchima renale determinano un accumulo eccessivo di proteine della matrice extracellulare (per esempio, collagene IV, laminina) che, incrementando le dimensioni della massa fibrotica, danneggiano irreversibilmente il tessuto renale portando alla disfunzione dell'organo $(5,6)$. Pertanto, l'accurata comprensione di questo processo e la potenziale modulazione farmacologica di elementi chiave biologico-molecolari coinvolti nella fibrosi rappresenta un bersaglio importante per la ricerca in campo nefrologico.

In questo contesto, lo studio approfondito della fisiologia delle cellule tubulari renali sta assumendo un'importanza sempre maggiore. Una serie di autori ha ipotizzato che il meccanismo patogenetico alla base della fibrosi interstiziale abbia inizio anche nel compartimento tubulare e si aggiunga a tutti i processi innescati dall'attivazione di elementi interstiziali e/o dalle alterazioni secondarie glomerulari. Infatti, la fonte dei mio-fibroblasti è molteplice e include: la migrazione tessutale dei fibrociti circolanti, la differenziazione di fibroblasti locali o periciti e la trasformazione diretta di cellule endoteliali (transizione endotelio-mesenchimale) o epiteliali (transizione epitelio-mesenchimale) nel fenotipo mio-fibroblastico (7). In particolare, durante il processo di transizione epitelio-mesenchimale (EMT) le cellule epiteliali diventano mobili e subiscono un incremento significativo di markers di superficie di natura mesenchimale come Vimentina (VIM) e actina muscolare liscia (SMA) $(8,9)$. In aggiunta, grazie al rimodellamento del citoscheletro, alla secrezione delle metalloproteinasi della matrice (MMP) e alla degradazione della membrana basale, le cellule con acquisito fenotipo miofibroblastico sono in grado di migrare nell'interstizio dove svolgono un ruolo chiave nel processo patogenetico che porta al danno renale cronico (10).

Tale assunto è stato confermato da numerosi studi in vivo effettuati su modelli animali di nefropatie croniche e in preparati istologici prelevati da pazienti affetti da nefropatia diabetica (ND) e altre forme patogenetiche progressive $(11,12)$.

L'EMT si verifica in risposta a diversi stimoli: a) ipossia; b) stress-ossidativo (meccanismo mediato principalmente dalle specie reattive dell'ossigeno); c) azione tossica mediata dai prodotti di avanzata glicazione delle proteine (AGE); d) cambio fenotipico mediato dalle MMP; e) intervento di markers di infiammazione acuta e cronica (per esempio l'IL-1) e fattori di crescita profibrotici come transforming growth factor-beta (TGF-beta), connettive tissue growth factor (CTGF) e fibroblast growth factor-2 (FGF-2) (13).

\section{Fibrosi e transizione epitelio-mesenchi- male: ruolo dell'eparanasi}

Nel tentativo di comprendere meglio la complessa macchina biologica associata alla genesi del danno cronico renale, negli ultimi anni, sono stati effettuati, una serie di studi finalizzati all'individuazione di nuovi elementi coinvolti nella fibrogenesi e sono stati selezionati vari biomarker di progressione e potenziali target di intervento terapeutico. Tra questi, l'eparanasi (HPSE) sembra essere un fattore di indubbio interesse e degno di esteso approfondimento.

Questo enzima è un endo- $\beta$-D-glucuronidasi che taglia le catene dell'eparan solfato (HS) a livello di specifici siti intracatena, generando frammenti di circa 5-7 kDa. Tagliando le catene di HS, l'eparanasi partecipa alla degradazione e al rimodellamento della matrice extracellulare (ECM) (14).

Tale enzima è primariamente espresso a livello della placenta, cheratinociti, piastrine e cellule attivate del sistema immune. La sua iper-espressione è stata osservata in un gran numero di tumori umani e conferisce ad essi un fenotipo più invasivo. In aggiunta, è stato dimostrato che tale enzima è coinvolto nella neovascolarizzazione, nell'infiammazione e nell'autoimmunità, sostenendo la migrazione delle cellule endoteliali vascolari e delle cellule del sistema immunitario (15).

Data la varietà di molecole associate all'HS sulla superficie cellulare e a quelle della ECM, è plausibile che l'attività e la biodisponibilità dell'eparanasi debba essere altamente modulata. Un primo controllo avviene a livello trascrizionale ma poi prosegue a livello post-traduzionale in un insieme di eventi che prendono il nome di processamento dell'enzima. Anche la localizzazione cellulare e i meccanismi di secrezione intervengono nella regolazione (16).

L'espressione dell'eparanasi è regolata positivamente 


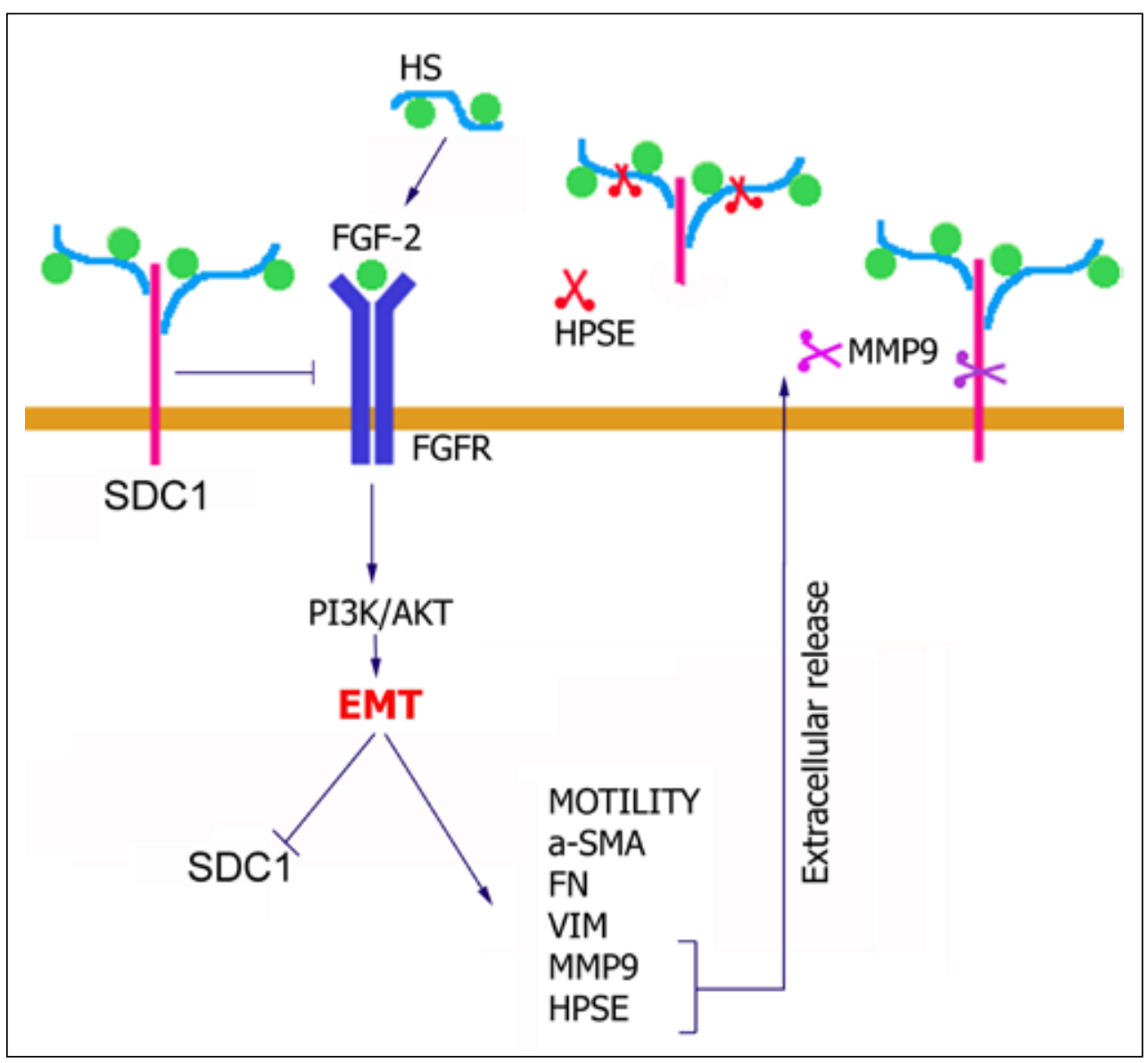

Fig. 1 - Meccanismo cellulare Eparanasi-mediato associato alla Transizione Epitelio-Mesenchimale (EMT) renale.

da fattori di trascrizione come Sp1, GA-binding protein (GABP), Ets1, Ets2, early growth response-1 (EGR1) e negativamente dal fattore di trascrizione p53 e dalla metilazione del DNA. L'eparanasi è, inoltre, modulata da varie molecole endogene: elastasi, attivatore tissutale del plasminogeno, trombina, alti livelli di glucosio, angiotensina II ed aldosterone, radicali liberi, citochine pro-infiammatorie, acidi grassi e fattore di necrosi tissutale-alfa (TNF-alfa), Vascular endothelial growth factor (VEGF) ed estrogeni $(17,18)$.

La degradazione dei vari costituenti dell'ECM, inclusi i proteoglicani eparan-solfato (HSPG) favorisce il rilascio di fattori trofici legati all'HS quali Fibroblast growth factor-2 (FGF-2) e VEGF.

Studi recenti hanno, inoltre, evidenziato che l'aumentata espressione dell'eparanasi correla positivamente con i livelli di fosforilazione della fosfatidilinositolo-3-kinasi (PI3K) e AKT e la somministrazione esogena di eparanasi in cellule endoteliali stimola la fosforilazione di AKT. Questi dati suggeriscono la presenza di recettori di superficie per l'eparanasi (16).

Nonostante ciò, è evidente che i meccanismi molecolari responsabili della iper-espressione dell'eparanasi riman- gono tuttora parzialmente sconosciuti.

Dal punto di vista meramente clinico, il ruolo dell'eparanasi è stato ben delineato in fenomeni quali metastasi, angiogenesi e infiammazione e dati recenti ne individuano un ruolo anche in varie nefropatie proteinuriche.

Studi in vivo, sia su modelli sperimentali animali (danno renale da steptozoicina, nefropatia da adriamicina, nefrosi indotta da puromicina, nefrite di Heymann, lupus e nefropatia diabetica) che report clinici/istopatologici effettuati sull'uomo (nefropatia diabetica, glomerulonefrite a lesioni minime, glomerulonefrite membranosa, glomerulonefrite a depositi mesangiali di IgA e nefrite lupica) rivelano un'aumentata espressione renale del suddetto enzima (19).

Alcuni studi iniziali sottolineano che livelli elevati e patologici di eparanasi a livello della struttura glomerulare sono in grado di causare proteinuria attraverso la digestione dei proteoglicani dell'HS della membrana basale glomerulare determinando di conseguenza un'alterazione funzionale renale. Risulta infatti emergere, in tali soggetti, un sistema alterato di ancoraggio di vari fattori trofici come FGF-2, VEGF e connective tissue growth factor (CTGF) (18). 
Negli ultimi anni accanto all'ipotesi patogenetica eparanasi-mediata glomerulare è emerso un nuovo interesse sul ruolo di questo enzima a livello tubulare $(20,21)$.

\section{Eparanasi e aspetti clinici}

In un recente lavoro pubblicato dal nostro gruppo è stato chiaramente dimostrato che, vista la presenza costitutiva dell'eparanasi sulle cellule epiteliali tubulari umane, questo enzima possa avere un ruolo fisiologico nel mantenimento dell'omeostasi cellulare, nella costituzione e nel mantenimento della matrice extracellulare e che l'alterazione di questo equilibrio sia alla base dell'instaurarsi di importanti meccanismi patogenetici responsabili del danno cronico in molte patologie renali (22).

In particolare è emerso che pazienti affetti da nefropatia diabetica (ND) avevano elevati livelli tubulari di eparanasi e la sua attività era positivamente correlata alla secrezione urinaria di alfa 2-microglobulina (21) e negativamente associata ai livelli di HS (20). Questi risultati sottolineavano il possibile utilizzo in futuro di tale proteina come biomarker di danno tubulo-interstiziale da utilizzare routinariamente in ambito nefrologico.

L'esposizione delle cellule tubulari ad alti livelli di glucosio, albumina e prodotti di glicazione avanzata (AGE) porta all'attivazione di vari segnali intracellulari con conseguente alterazione nell'espressione genica di fattori di crescita e citochine, una anormale sintesi e degradazione delle proteine che costituiscono la matrice extracellulare (23) e alla sintesi di eparanasi da parte dei tubulociti. L'eparanasi a sua volta è responsabile della degradazione dell'HS e della regolazione del Sindecano-1 (SDC1), il proteoglicano dell'HS più abbondante a livello di cellule tubulari. Ciò genera la condizione profibrotica tipica della MRC.

Complessivamente la sovra-espressione dell'eparanasi nelle cellule tubulari, indotta da stimoli diabetici, comporta un rimodellamento dell'HS e del SDC-1 creando un'alterazione nella disponibilità dei fattori di crescita, come FGF-2 e TGF-beta, che possono innescare la fibrosi tubulare-interstiziale.

Abbiamo inoltre recentemente dimostrato che l'eparanasi è necessaria all'FGF-2 per produrre la transizione epitelio-mesenchima (24). In particolare, l'FGF-2 induce EMT nelle cellule tubulari aumentando l'espressione dei marcatori mesenchimali alfa-SMA, VIM e fibronectina $(\mathrm{FN})$, porta alla degradazione della membrana basale mediante la secrezione di MMP9 ed aumenta la motilità cellulare. Tali eventi non sono presenti in cellule tubulari private dell'eparanasi.

Ciò può essere spiegato dal fatto che cellule prive dell'e- paranasi esprimono quantità maggiori del SDC-1 che inibisce fisiologicamente l'FGF-2 quando presente sulla superficie cellulare. L'FGF-2 induce EMT mediante la via di trasduzione del segnale PI3K/AKT e produce nelle cellule tubulari un circuito autocrino per sostenere il proprio segnale attraverso molteplici meccanismi: a) riduce l'espressione del SDC-1; b) aumenta l'espressione ed il rilascio di MMP9, una metalloproteasi in grado di tagliare il dominio extra-citoplasmatico del SDC-1 e c) incrementa l'espressione e l'attività dell'eparanasi che è in grado di convertire i frammenti solubili di SDC-1-HS da inibitori a potenti attivatori dell'FGF-2 (24).

Un'importante conferma a queste teorie è stata recentemente fornita da Gil e coll (25), i quali hanno dimostrato che il topo-KO per l'eparanasi non sviluppa la nefropatia diabetica indotta da streptozoicina. In particolare $\mathrm{i}$ topi Hpse-KO non sviluppano proteinuria, l'espressione urinaria di albumina rimane normale e soprattutto presentano normali livelli di espressione della matrice mesangiale, assenza di infiltrazione macrofagica e inconsistente fibrosi tubulo-interstiziale.

Pertanto visto l'importante ruolo di questo enzima sono state messe a punto numerose strategie per inibire la sua espressione genica e/o l'attività enzimatica. E visto il suo coinvolgimento in numerose nefropatie e nell'induzione della fibrosi tubulare esso rappresenta un importante target farmacologico. Infatti, sono in fase di studio inibitori dell'eparanasi (in particolar modo eparinoidi, glicosaminoglicani) soprattutto da impegnare nel trattamento di forme progressive o avanzate di danno renale (26).

Comunque, è importante che la ricerca di base e clinica aggiunga ulteriori tasselli nella comprensione del meccanismo intracellulare mediato da questo enzima per standardizzare il suo utilizzo nella comune pratica clinico-laboratoristica.

\section{Riassunto}

Il trattamento poli-farmacologico ha determinato, nel corso degli anni, un significativo rallentamento della progressione della malattia renale cronica verso lo stadio di uremia terminale, ma siamo ancora distanti dallo sviluppo di interventi terapeutici in grado di bloccare questo inesorabile e irreversibile processo. Studi clinico-patologici hanno chiaramente dimostrato che il principale elemento coinvolto nel danno renale è la fibrosi tubulo-interstiziale e che il meccanismo patogenetico alla base di questa condizione ha inizio in larga parte nel compartimento tubulare. In particolare, il processo di transizione epitelio-mesenchimale gioca un ruolo im- 
portante nella genesi del danno cronico. Durante questo processo, le cellule epiteliali tubulari subiscono un incremento significativo di markers di superficie di natura mesenchimale e, grazie al rimodellamento del citoscheletro e alla degradazione della membrana basale, sono in grado di migrare nell'interstizio dove svolgono un ruolo chiave nel processo patogenetico. In questo contesto, sembra avere un ruolo chiave l'enzima eparanasi, una endo- $\beta$-D-glucuronidasi che taglia le catene dell'eparansolfato a livello di siti specifici intracatena, e partecipa attivamente alla degradazione e al rimodellamento della matrice extracellulare. La degradazione dei vari costituenti dell'ECM, inclusi i proteoglicani eparan-solfato favorisce il rilascio di fattori trofici quali il FGF-2 che induce l'espressione dei marcatori mesenchimali alfa-SMA, VIM e FN, porta alla degradazione della membrana basale mediante la secrezione di metalloproteinasi della matrice ed aumenta la motilità cellulare. L'epressione dell'eparanasi è regolata da fattori di trascrizione, dalla metilazione del DNA e da varie molecole endogene. L'importanza di questo enzima è stata confermata clinicamente dal riscontro di una sua iperespressione in preparati istologici di biopsie effettuate in soggetti affetti da nefropatie croniche (per esempio, nefropatia diabetica). Pertanto visto l'importante ruolo dell'eparanasi sono in fase di standardizzazione numerose strategie per inibire la sua espressione genica e/o la sua attività enzimatica. Infine, è stato proposto il suo possibile utilizzo come biomarker di progressione del danno tubulo-interstiziale da utilizzare routinariamente in ambito nefrologico.

Parole Chiave. Fibrosi, Insufficienza renale cronica, Transizione epitelio-mesenchimale, Nefrologia

Conflitti di interesse: nessuno

Contributi economici agli autori: nessuno

Indirizzo degli Autori:

Dr. Gianluigi Zaza, MD, PhD

U.O.C. Nefrologia e Dialisi

Azienda Ospedaliera Universitaria Integrata

Piazzale A. Stefani 1

37126 Verona (VR)

gianluigi.zaza@univr.it

\section{Bibliografia}

1. Castro AF, Coresh J. CKD surveillance using laboratory data from the population-based National Health and $\mathrm{Nu}-$ trition Examination Survey (NHANES). Am J Kidney Dis 2009; 53(Suppl): S46-55.

2. Turner JM, Bauer C, Abramowitz MK, Melamed ML, Hostetter TH. Treatment of chronic kidney disease. Kidney Int 2012; 81: 351-62.

3. Hewitson TD. Renal tubulointerstitial fibrosis: Common but never simple. Am J Physiol Renal Physiol 2009; 296: F1239-44.

4. Liu Y. Renal fibrosis: New insights into the pathogenesis and therapeutics. Kidney Int 2006; 69: 213-7.

5. Liu Y. Epithelial to mesenchymal transition in renal fibrogenesis: Pathologic significance, molecular mechanism, and therapeutic intervention. J Am Soc Nephrol 2004; 15: 1-12.

6. Mason RM, Wahab NA. Extracellular matrix metabolism in diabetic nephropathy. J Am Soc Nephrol 2003; 14: 1358-73.
7. Hinz B, Phan SH, Thannickal VJ, et al. Recent developments in myofibroblast biology: paradigms for connective tissue remodeling. Am J Pathol 2012; 180(4): 1340-55.

8. Iwano M, Plieth D, Danoff TM, Xue C, Okada H, Neilson EG. Evidence that fibroblasts derive from epithelium during tissue fibrosis. J Clin Invest 2002; 110: 341-50.

9. Zeisberg EM, Potenta SE, Sugimoto H, Zeisberg M, Kalluri R. Fibroblasts in kidney fibrosis emerge via endothelial-to-mesenchymal transition. J Am Soc Nephrol 2008; 19: 2282-7.

10. Strutz FM. EMT and proteinuria as progression factors. Kidney Int 2009; 75: 475-81.

11. Burns WC, Twigg SM, Forbes JM, et al. Connective tissue growth factor plays an important role in advanced glycation end product-induced tubular epithelial-to-mesenchymal transition: Implications for diabetic renal disease. J Am Soc Nephrol 2006; 17: 2484-94.

12. Liu Y. New insights into epithelial-mesenchymal transition in kidney fibrosis. J Am Soc Nephrol 2010; 21: 212-22.

13. Lan HY. Tubular epithelial-myofibroblast transdifferentiation mechanisms in proximal tubule cells. Curr Opin 
Nephrol Hypertens 2003; 12: 25-9.

14. Ilan N, Elkin M, Vlodavsky I. Regulation, function and clinical significance of heparanase in cancer metastasis and angiogenesis. Int J Biochem Cell Biol 2006; 38: 2018-39.

15. Parish CR, Freeman C, Hulett MD. Heparanase: A key enzyme involved in cell invasion. Biochim Biophys Acta 2001; 1471: M99-108.

16. Nasser NJ. Heparanase involvement in physiology and disease. Cell Mol Life Sci 2008; 65: 1706-15.

17. Vreys V, David G. Mammalian heparanase: What is the message? J Cell Mol Med 2007; 11: 427-52.

18. Szymczak M, Kuzniar J, Klinger M. The role of heparanase in diseases of the glomeruli. Arch Immunol Ther Exp (Warsz) 2010; 58: 45-56.

19. van den Hoven MJ, Rops AL, Vlodavsky I, Levidiotis V, Berden JH, van der Vlag J. Heparanase in glomerular diseases. Kidney Int 2007; 72: 543-8.

20. van den Hoven MJ, Rops AL, Bakker MA, et al. Increased expression of heparanase in overt diabetic nephropathy. Kidney Int 2006; 70: 2100-108.
21. Rops AL, van den Hoven MJ, Veldman BA, et al. Urinary heparanase activity in patients with type 1 and type 2 diabetes. Nephrol Dial Transplant 2011

22. Masola V, Gambaro G, Tibaldi E, Onisto M, Abaterusso C, Lupo A. Regulation of heparanase by albumin and advanced glycation end products in proximal tubular cells. Biochim Biophys Acta 2011

23. Pollock CA, \& Poronnik P. Albumin transport and processing by the proximal tubule: Physiology and pathophysiology. Curr Opin Nephrol Hypertens 2007; 16: 359-64.

24. Masola V, Gambaro G, Tibaldi E, et al. Heparanase and syndecan-1 interplay orchestrates fibroblast growth factor-2-induced epithelial-mesenchymal transition in renal tubular cells. J Biol Chem 2012; 287: 1478-88.

25. Gil N, Goldberg R, Neuman T, et al. Heparanase is essential for the development of diabetic nephropathy in mice. Diabetes 2012; 61: 208-16.

26. Gambaro G, Kong NC. Glycosaminoglycan treatment in glomerulonephritis? an interesting option to investigate. J Nephrol 2010; 23: 244-52. 